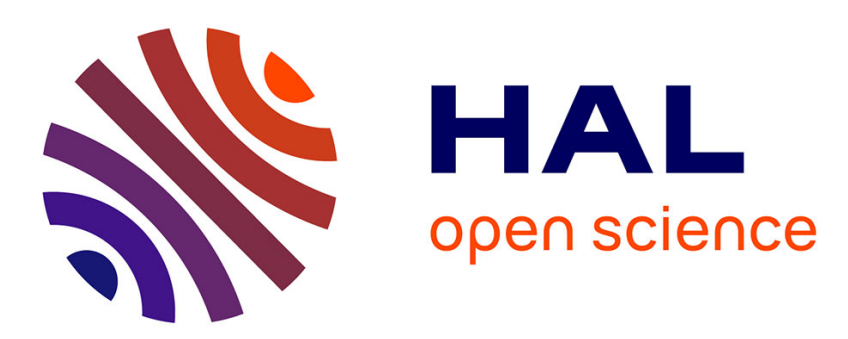

\title{
Fluorescence diffuse optical tomography: Time-resolved versus continuous-wave in the reflectance configuration
}

Nicolas Ducros, Cosimo d'Andrea, Andrea Bassi, Françoise Peyrin

\section{To cite this version:}

Nicolas Ducros, Cosimo d'Andrea, Andrea Bassi, Françoise Peyrin. Fluorescence diffuse optical tomography: Time-resolved versus continuous-wave in the reflectance configuration. Innovation and Research in BioMedical engineering, 2011, 32 (4), pp.243 - 250. 10.1016/j.irbm.2011.04.001 . hal00875236

\section{HAL Id: hal-00875236 \\ https://hal.science/hal-00875236}

Submitted on 21 Oct 2013

HAL is a multi-disciplinary open access archive for the deposit and dissemination of scientific research documents, whether they are published or not. The documents may come from teaching and research institutions in France or abroad, or from public or private research centers.
L'archive ouverte pluridisciplinaire HAL, est destinée au dépôt et à la diffusion de documents scientifiques de niveau recherche, publiés ou non, émanant des établissements d'enseignement et de recherche français ou étrangers, des laboratoires publics ou privés. 


\title{
Fluorescence diffuse optical tomography: time-resolved versus continuous-wave in the reflectance configuration
}

\author{
N. Ducros ${ }^{\mathrm{a}, *}$, C. D’Andrea ${ }^{\mathrm{a}, \mathrm{b}}$, A. Bassi ${ }^{\mathrm{a}}$, F. Peyrin ${ }^{\mathrm{c}}$ \\ ${ }^{a}$ Instituto di Fotonica e Nanotecnologie (IFN-CNR) ; Dipartimento di Fisica, Politecnico di Milano, Piazza Leonardo da Vinci 32, I-20133 Milan, Italy \\ ${ }^{b}$ Italian Institute of Technology (IIT), Piazza Leonardo da Vinci 32, I-20133 Milan, Italy \\ ${ }^{c}$ CREATIS-LRMN, CNRS; INSERM; Université de Lyon I; INSA de LYON, Villeurbanne, France.
}

\begin{abstract}
Context and objectives- In the context of fluorescence diffuse optical tomography, in the transmittance configuration, the time-resolved approach was shown to improve the reconstruction quality compared to the continuous-wave approach but this improvement seriously decreases in the presence of noise. In this paper the additional value of time-resolved approach is investigated in the reflectance configuration.

Material and methods- A comparative study between time-resolved and continuous-wave reconstructions is provided in the reflectance configuration. Reconstructions are performed from synthetic measurements in a slab geometry assuming Poisson noise statistics.

Results and discussion - In the reflectance configuration, the reconstruction quality, expressed in terms of the global reconstruction error or in terms of the localization and quantification of a local inclusion, is shown to be sensibly higher when the time-resolved approach is considered rather than the continuous-wave one. This behavior is observed for maximum numbers of detected photons as low as $10^{4}$.

Conclusion- In the reflectance configuration, considering realistic level of signal, the time-resolved approach is shown to outperform continuous-wave approach.
\end{abstract}

Keywords: Fluorescence diffuse optical tomography, time-resolved approach, turbid media, 3D reconstructions, reflectance, transmittance.

\section{Résumé}

Contexte et objectif - Dans le cadre de la tomographie optique diffuse de fluorescence, l'approche temporelle permet une amélioration de la qualité de reconstruction par rapport à l'approche en continu plus classique. Cette amélioration reste cependant limitée en présence de bruit. Cette observation vaut lorsque le milieu est étudié en transmission. L'objectif du présent article est d'évaluer l'apport du temporel par rapport au continu lorsque le milieu est étudié en réflexion.

Matériel et méthodes - Nous présentons une étude comparative entre l'approche temporelle et l'approche continue pour une acquisition en réflexion. Les reconstruction sont réalisées à partir de mesures simulées dans un milieu parallélépipédique, considérant un bruit de Poisson.

Résultats et discussion - En réflexion, la qualité de reconstruction est sensiblement meilleure pour l'approche temporelle que pour l'approche continue, que la qualité de reconstruction soit exprimée en termes d'erreur de reconstruction globale ou bien en termes de localisation et de quantification d'une inclusion locale. Ce comportement est observé pour un nombre maximum de photons détecté se réduisant jusqu'à $10^{4}$.

Conclusion-Dans des scénarios réalistes, pour lesquels le nombre maximum de photons détectés est de l'ordre de $10^{6}$, l'approche temporelle surpasse en performance l'approche en continu pour une acquisition en réflexion, alors que les deux approches présentent des prestations similaires pour une acquisition en transmission.

Mots-clefs : Tomographie optique de fluorescence, approche temporelle, milieux diffusants, reconstructions 3D, réflection, transmission.

\footnotetext{
*. Corresponding author

Email address: nicolas.ducros@polimi.it (N. Ducros)
} 


\section{Introduction}

In the near-infrared region (NIR), the absorption of biological tissues is low enough to allow photons for propagating several centimeters. This enables to localize optical heterogeneities embedded in thick media [1]. By employing a setup comprising a set of external light sources and detectors, the optical properties of a tissue can be recovered by applying the principles of tomography. In this context, the aim of diffuse optical tomography (DOT) is to provide three-dimensional (3D) maps of the absorption and scattering coefficients of a tissue [2, 3]. More recently, the development of NIR fluorescent markers such as Indocyanine Green (ICG) has led to a novel technique, called fluorescence DOT (FDOT) or fluorescence molecular tomography (FMT), which is capable of determining the 3-D local concentrations of fluorescent markers $[4,5,6]$. New markers can tag specific proteins, acting as molecular probes. The so-called active probes are designed to exhibit fluorescence only in the vicinity of an enzyme cleaving a peptide [7]. The development of quantum dots is also promising to enlarge the range of fluorescent markers [8]. In this context, FDOT can play a significant role in small-animal imaging as well as in drug discovery.

FDOT approaches can be broadly classified into three groups that differ in the type of excitation and detection. Continuouswave $(\mathrm{CW})$ FDOT is based on the measurement of the attenuation of a steady state excitation light [9, 10, 11, 12]. Frequency-domain (FD) FDOT is based on the measurement of the phase and demodulation of an amplitude-modulated excitation [13]. Time-resolved (TR) FDOT exploits the measurement of the temporal distortion of an excitation light pulse $[14,15,16,17,18,19,20]$. FD and TR provide significant advantages with respect to $\mathrm{CW}$ approach. In particular, they allow to separate absorption and diffusion contributions [21], to derive fluorescence lifetime [22] and more generally to provide a data set with a higher information content which can lead to better reconstruction of deeply-embedded markers [23]. This last aspect, is particularly relevant for TR measurements, which contain any modulation frequency, while FD measurements can only be performed, in practice, for few of them.

Despite these remarks, CW approaches are the most frequently used to recover the marker concentration. This is partially due to instrumentation concerns, in fact, while the CW approaches are inexpensive and easy to develop, the FD and TR FDOT are more costly and requires careful handling. Moreover, this can be explained by a lack of comparative study that could set the domain of interest of both techniques. Although $\mathrm{CW}$ and TR are both long-standing approaches in FDOT, few studies have been dedicated to compare their reconstruction performances. In a recent work, we showed that the TR approach outperforms the CW approach in situations when a sufficient number of photons is collected [24]. However, this study was restricted to the transmittance configuration, i.e. for a medium lying between the sources and the detectors. Here, the performance of CW and TR FDOT are evaluated in the reflectance configuration, i.e. for sources and detectors lying on the same side of the medium. The reflectance configuration is quite relevant because in many applications (e.g. tissue oxymetry, brain function imaging and prostate tomography) it represents the only available geometry. Moreover, reflectance geometry can better exploit the information content of TR data set, because the information of the inclusion depth is encoded into the time of flight [17].

The aim of this paper is to compare the quality of reconstruction in transmission and reflexion time-resolved acquisitions.

In section 2 we introduce the basic theory of light propagation as well as the modeling of the forward and inverse problems. Section 3 describes the methods used for the simulation of TR measurements and for reconstructions. Finally section 4 presents the results.

\section{Theory}

\subsection{Light propagation}

Light propagation within biological tissues is dominated by absorption and scattering. Such media are commonly referred to as turbid media. A variety of theoretical models accounting for the propagation of light within such media have been developed. Among them the deterministic models are based on the resolution of a partial derivative equation deriving from the radiative transfer equation (see Sec. 3 of [25] for an overview). Although more general models are available, the light propagation in FDOT is classically assumed to follow the diffusion equation. Within this framework, the propagation operator acting on the photon density photons $\phi$ at position $\mathbf{r}$ and time $t$ is given by:

$$
\mathcal{P}=-\nabla \frac{1}{3 \mu_{s}^{\prime}} \nabla+\frac{1}{v} \frac{\partial}{\partial t}+\mu_{a}
$$

where $\mu_{a}$ is the absorption coefficient, $\mu_{s}^{\prime}$ is the reduced scattering coefficient, and $v$ is the speed of light within the medium.

\subsection{FDOT equations}

The principle of FDOT is depicted in Fig. 1. After illumination of the medium at position $\mathbf{s}$, the photon density $\phi_{x}$ propagates at wavelength $\lambda_{x}$ within the medium. It is absorbed by the fluorescent markers that act as secondary sources by re-emitting light at a higher wavelength $\lambda_{f}>\lambda_{x}$. Mathematically, this leads to the following system of coupled equations [26]:

$$
\left\{\begin{array}{l}
\mathcal{P} \phi^{x}(\mathbf{r}, t)=\delta(\mathbf{r}-\mathbf{s}, t), \\
\mathcal{P} \phi^{m}(\mathbf{r}, t)=\left[\phi^{x}(\mathbf{r}, t) \star p(t)\right] c(\mathbf{r}) .
\end{array}\right.
$$

Where $\phi^{x}(r, t)$ and $\phi^{m}(r, t)$ are respectively the photon density at wavelength $\lambda_{x}$ and $\lambda_{m}$, and $c(\mathbf{r})$ is the local concentration of the fluorescent markers. Equation (2a) describes the propagation of light at wavelength $\lambda_{x}$, while Eq. (2b) describes the propagation of light at wavelength $\lambda_{m}$. Note that the source term of Eq. (2b) is the temporal convolution of the excitation photon density and pulse response of the fluorescence marker $p$. The latter is given by

$$
p(t)=\frac{\eta}{\tau} \exp \left(-\frac{t}{\tau}\right) \mathrm{d} V
$$




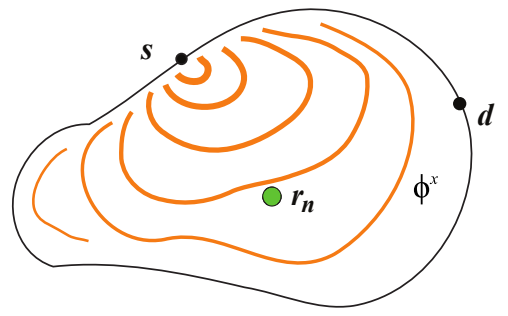

a)

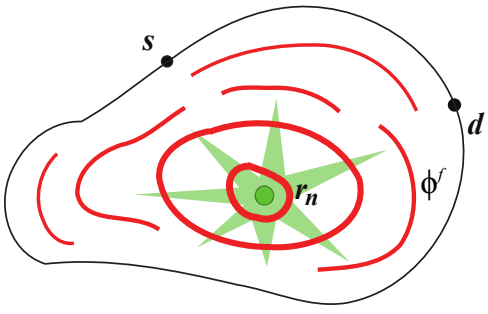

b)

Figure 1: Principle of FDOT. a) The medium is excited by at position $\mathbf{s}$ by a pulse of light at wavelength $\lambda_{x}$. b) Fluorescence light is emitted at wavelength $\lambda_{f}>\lambda_{x}$ by a fluorophore located at position $\mathbf{r}$.
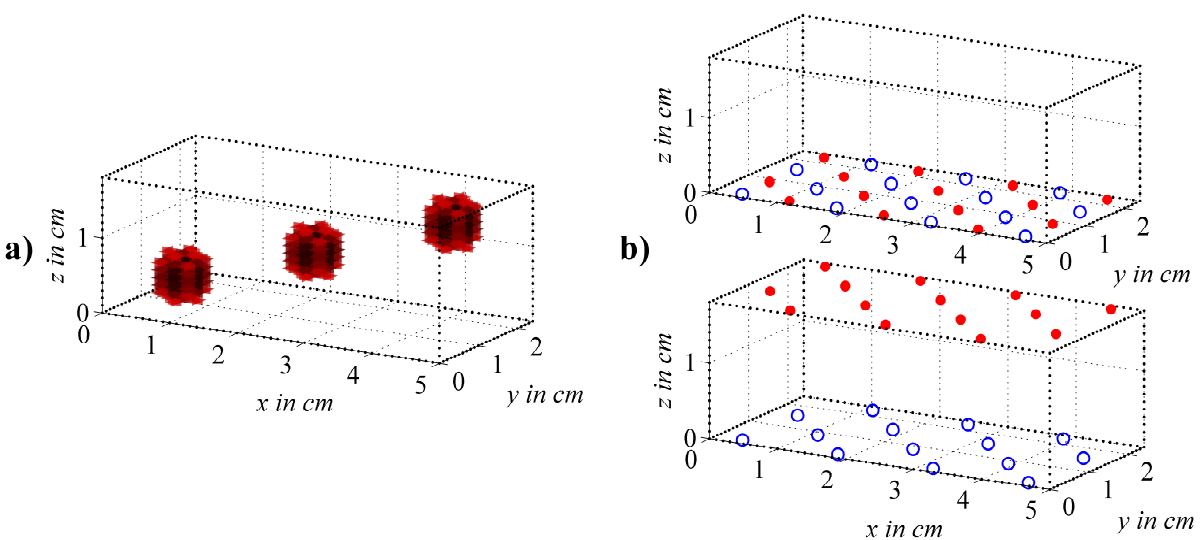

Figure 2: a) Numerical phantom. The red dots represent the source points, the blue rings the detector points, and the thin black dots the mesh nodes (for clarity, only those at the border are represented). The optical properties of the medium are the following: $\mu_{a}=0.1 \mathrm{~cm}^{-1}, \mu_{s}^{\prime}=10 \mathrm{~cm}^{-1}, n=1.4$, and $\tau=0.3 \mathrm{~ns}$. b) Source-detector configuration. On top is represented the reflectance configuration, in the bottom the transmittance configuration.

where $\tau$ is the fluorescence lifetime of the marker, $\eta$ its efficiency, and $c(\mathbf{r})$ its local concentration.

As recommended in [27], the photons density $\phi^{m}$ is assumed to be the quantity measured by the acquisition set-up.

\subsection{Forward problems}

Let $\left\{\mathbf{s}_{i}\right\}, i \in\{1, \ldots, I\}$ be the set of source points and $\left\{\mathbf{d}_{j}\right\}, j \in$ $\{1, . ., J\}$ the set of detection points. The medium is discretized into $\mathrm{N}$ voxels centered in $\left\{\mathbf{r}_{n}\right\}$. We note $m_{i, j}$ the quantity of light detected at position $\mathbf{d}_{j}$ after illumination at position $\mathbf{s}_{i}$ and $c_{n}$ the marker concentration into the voxel centered in $\mathbf{r}_{n}$. FDOT is an intrinsically linear problem of the form [26]

$$
\mathbf{m}(t)=\mathbf{W}(t) \mathbf{c},
$$

where $\mathbf{c}=\left[c_{1}, . ., c_{N}\right]^{T}$ is the marker concentration vector, $\mathbf{m}(t)=\left[m_{1,1}(t), m_{1,2}(t), . ., m_{I, J}(t)\right]^{T}$ is the measurement vector, and $\mathbf{W}(t)$ is the weight matrix. The entries of $\mathbf{W}(t)$ for each couple of source $\mathbf{s}_{i}$ and detector $\mathbf{d}_{j}$ are given by

$$
w_{n}^{i, j}(t)=g\left(\mathbf{s}_{i}, \mathbf{r}_{n}, t\right) \star p(t) \star g\left(\mathbf{d}_{j}, \mathbf{r}_{n}, t\right) .
$$

where $g$ stands for the Green's functions of the propagation operator $\mathcal{P}$.

The use of extended source patterns together with extended detection patterns have also been introduced [28, 29, 30, 31].However, the framework defined in Eq. (4) and Eq. (5) remains identical.

\subsection{Inverse problem}

The inverse problem, which consists in finding c from Eq. (4), being ill-posed, a Tikhonov regularization scheme is used to solve it. For a forward problem of the form $\mathbf{y}=\mathbf{A c}$, the following Tikhonov solution $\mathbf{c}^{*}$ is considered

$$
\mathbf{c}^{*}=\underset{\mathbf{c}}{\arg \min }\|\mathbf{y}-\mathbf{A c}\|_{\mathbf{Q}}^{2}+\alpha\|\mathbf{c}\|^{2}
$$

where $\alpha$ is the regularization parameter. To enable comparison between solutions obtained from different system matrix $\mathbf{A}$ and measurement vector $\mathbf{y}$, the regularization parameter is chosen so as to set the variability of the solution to a prescribed value, i.e few percents (details are provided in [19]). 


\section{Method}

\subsection{Numerical phantom}

We consider the parallelepiped of size $5 \times 2.5 \times 1.8 \mathrm{~cm}^{3}$ depicted in Fig. 2a. The absorption coefficient $\mu_{a}$ is set to $0.1 \mathrm{~cm}^{-1}$, the scattering coefficient $\mu_{s}^{\prime}$ to $10 \mathrm{~cm}^{-1}$, and the refractive index $n$ to 1.4. The fluorescent marker we considered has a lifetime $\tau$ of $0.7 \mathrm{~ns}$ (Alexa 750). The markers concentrate to form three inclusions centered in $\mathbf{r}_{1}=[0.9,0.5,0.5] \mathrm{cm}, \mathbf{r}_{2}=[2.5,1.1,0.9]$ $\mathrm{cm}$, and $\mathbf{r}_{3}=[4.1,1.9,1.3] \mathrm{cm}$. At the center of each inclusion the marker concentration is set to $1 \mu \mathrm{M}$. Away from the center the marker concentration decays gradually to $0 \mu \mathrm{M}$.

\subsection{Source-detector configurations}

The medium is illuminated in $S=14$ source points, and light is collected at $D=13$ detection points. The sources and detectors are uniformly arranged onto two grids of size $4.2 \times 1.8 \mathrm{~cm}^{2}$ as depicted in Fig. 2 b.

In this study, two source-detector configurations are investigated, namely the transmittance and the reflectance configurations. In the transmittance configuration, sources and detectors are arranged into two distinct planes that surround the medium. In this case, the sources are placed in the plane $z=0 \mathrm{~cm}$ and the detectors in the plane $z=1.8 \mathrm{~cm}$. In the reflectance configuration, however, sources and detectors are arranged into the same plane, here the plane $z=0 \mathrm{~cm}$.

\subsection{Computation of the forward problem}

The computation of the forward problem consists in the calculation of the weight matrix $\mathbf{W}(t)$ whose entries are given by Eq. (5). The required Green's functions of Eq. (1) are obtained by means of the finite element Matlab package TOAST [32]. At this step, a grid of 24225 voxels of size $0.1 \times 0.1 \times 0.1 \mathrm{~cm}^{3}$ is considered and 128 time samples evenly distributed on the range $[0 ; 5.12]$ ns are calculated. Such fine grids are necessary to ensure the accuracy of the weight matrix. However, for the rest of the computation, a space grid of 5100 voxels of size $0.2 \times 0.2 \times 0.1 \mathrm{~cm}^{3}$ together with a time grid of 16 time windows of width $160 \mathrm{ps}$ ranging from $t=0.12 \mathrm{~ns}$ to $t=2.64 \mathrm{~ns}$ are considered.

As given by Eq. (4), the numerical measurements $\mathbf{m}(t)$ are obtained multiplying the weight matrix $\mathbf{W}(t)$ by the phantom concentration vector $\mathbf{c}_{\mathbf{p}}$.

\subsection{Instrument response function}

In order to simulate realistic conditions, the measurements are convolved by the instrument response function accounting for the experimental laser and detector. The following transformations are thus considered:

$$
\begin{aligned}
& m_{i, j}(t) \leftarrow m_{i, j}(t) \star f(t), \\
& w_{n}^{i, j}(t) \leftarrow w_{n}^{i, j}(t) \star f(t),
\end{aligned}
$$

where $f$ is the instrument response function accounting for the instrument temporal jitter. We considered the experimental instrument response function represented in Fig. 3. This stateof-the-art system consists of a mode-locked Ti:sapphire laser

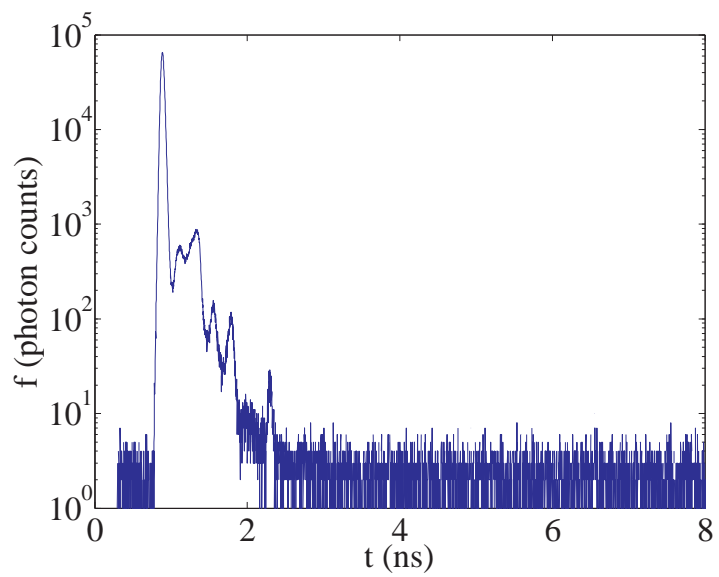

Figure 3: Instrument response function. The system consists of a mode-locked Ti:sapphire laser pumped by an argon laser and a cooled micro-channel plate photomultiplier [33].

(3900, Spectra Physics, USA) pumped by an argon laser (Innova 200, Coherent, USA) and a cooled micro-channel plate photomultiplier (R1564U, Hamamatsu, Japan) that offers good temporal resolution ( $<50 \mathrm{ps}, \mathrm{FWHM})$ [33].

\subsection{Noise model}

The measurement vector and weight matrix are both multiplied by the same constant allowing to account for the experiment integration time -the longer the integration time, the larger the number of detected photons. Here the multiplicative constant, noted $\kappa$, is chosen so as to set the maximum number of detected photons $N_{\max }$ to a prescribed value [24]:

$$
\max _{i, j} \kappa m_{i, j}=N_{\max } .
$$

The measurements are finally corrupted by Poisson noise. Mathematically, the noisy version $\widetilde{m}_{i, j}$ of the noiseless measurement $m_{i, j}$ is given by

$$
\widetilde{m}_{i, j}=P\left(\lambda=m_{i, j}\right),
$$

where $P(\lambda)$ denotes a Poisson distribution of mean $\lambda$. For any noiseless measurement $m_{i, j}$, a set 50 different noise realizations $\widetilde{m}_{i, j}$ is computed.

\subsection{Reconstruction approach}

Reconstructions are performed from both CW and TR measurements, leading to $\mathrm{CW}$ reconstructed concentration $\mathbf{c}_{c w}^{*}$ and the TR reconstructed concentration $\mathbf{c}_{t r}^{*}$. More precisely, $\mathbf{c}_{c w}^{*}$ is obtained by inverting the $\mathrm{CW}$ forward model:

$$
\mathbf{m}_{c w}=\mathbf{W}_{c w} \mathbf{c},
$$

where $\mathbf{m}_{c w}$ is the CW measurement vector and $\mathbf{W}_{c w}$ is the CW weight matrix. Equation (11a) derives from the integration of Eq. (4) over time, which leads to $\mathbf{m}_{c w}=\int \mathbf{m}(t) \mathrm{d} t$ and $\mathbf{W}_{c w}=$ $\int \mathbf{W}(t) \mathrm{d} t$. 

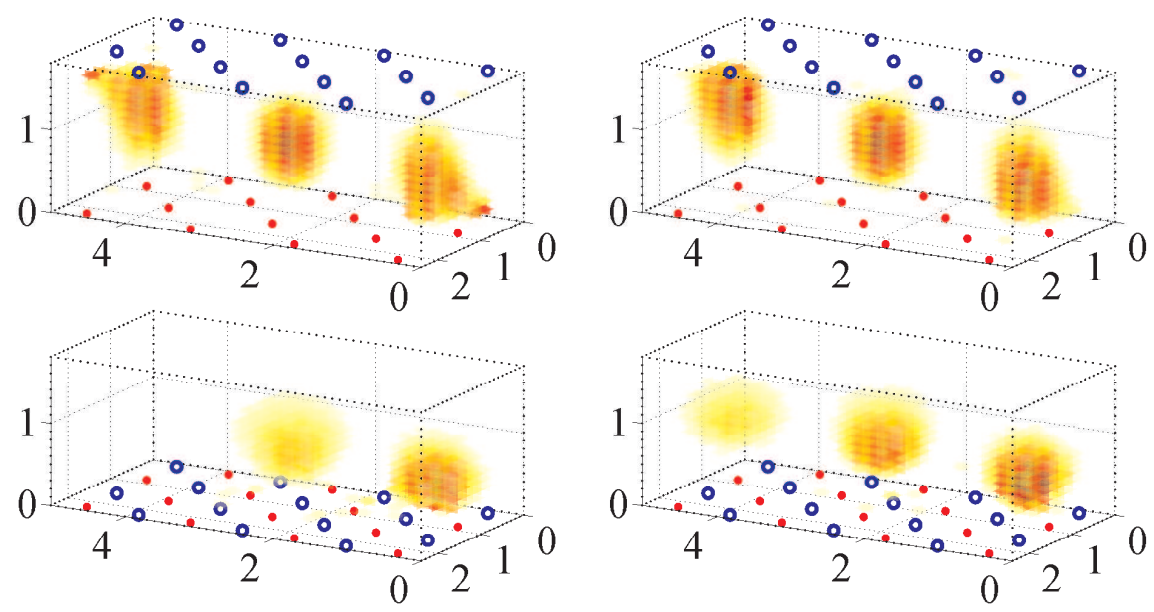

Figure 4: 3-D reconstructed marker concentration, for the CW modality (left-hand column) and the TR modality (right-hand column), in both the reflectance (bottom row) and the transmittance (top row) configurations. $N_{\max }$ is set to $10^{6}$ photons.

The TR reconstructed concentration $\mathbf{c}_{t r}^{*}$ results from the inversion of the TR forward model:

$$
\mathbf{m}_{t r}=\mathbf{W}_{t r} \mathbf{c},
$$

where $\mathbf{m}_{t r}$ is the TR measurement vector and $\mathbf{W}_{t r}$ is the TR weight matrix. Equation (11b) is obtained by concatenating the Eq. (4) evaluated at different times $\left\{t_{1}, . ., t_{K}\right\}$. This operation leads to $\mathbf{m}_{\text {tr }}=\left[\mathbf{m}\left(t_{1}\right), . ., \mathbf{m}\left(t_{K}\right)\right]^{T}$ and $\mathbf{W}_{t r}=\left[\mathbf{W}\left(t_{1}\right), . ., \mathbf{W}\left(t_{K}\right)\right]^{T}$.

\subsection{Performance metrics}

To quantify the reconstruction quality, we consider one global performance metric and two local ones. The two local performance metrics depend on the position the centers $\mathbf{r}_{i}^{*}$ of the reconstructed inclusions. The center positions $\mathbf{r}_{i}^{*}$ are determined searching the local maximum of $c^{*}$ closest to the groundtruth inclusion position $\mathbf{r}_{i}$. This simple approach turns out accurate enough not to involve segmentation method.

The reconstruction error $\mathcal{E}$. This global metric measures the distance between the phantom concentration $\mathbf{c}_{\mathbf{p}}$ and the reconstructed concentration $\mathbf{c}^{*}$. It is defined by

$$
\mathcal{E}=\frac{\left\|\mathbf{c}^{*}-\mathbf{c}_{\mathbf{p}}\right\|_{2}^{2}}{\left\|\mathbf{c}_{\mathbf{p}}\right\|_{2}^{2}}
$$

The reconstruction quality increases as the reconstruction error tends to 0 .

Localization error $\mathcal{L}$. This criterion assesses the ability of localizing an inclusion. It is defined as the distance between the center of the phantom inclusion $\mathbf{r}_{i}$ and the center of the reconstructed inclusion $\mathbf{r}_{i}^{*}$. Mathematically:

$$
\mathcal{L}=\left\|\mathbf{r}_{i}^{*}-\mathbf{r}_{i}\right\|_{2} .
$$

The reconstruction quality increases as the localization error tends to 0 .
Quantification $Q$. This criterion assesses the ability of quantifying the concentration of an inclusion. It is defined as the ratio between the concentration of the reconstructed inclusion and the concentration of the phantom inclusion, i.e.

$$
Q=\frac{c^{*}\left(\mathbf{r}_{i}^{*}\right)}{c_{p}\left(\mathbf{r}_{i}\right)} .
$$

The reconstruction quality increases as the quantification tends to 1 .

\section{Results and discussion}

In this section, we present reconstructions and their associated performance metrics for different values of the maximum number of detected photons $N_{\max }$. The larger $N_{\max }$ is -in practice the longer the integration time, the better the signal-to-noise ratio. The influence of $N_{\max }$ is of particular interest since the reconstruction quality mainly depends on this parameter [24].

First, a visual comparison between $\mathrm{CW}$ and TR reconstructions for both transmittance and reflectance configurations is provided in Fig. 4. In this figure, the maximum number of detected photons $N_{\max }$ is set to $10^{6}$ photons, which roughly corresponds to the largest value obtainable in experimental scenarios. Comparing the first row and the second row of Fig. 4, it can be seen that the reflectance configuration leads to reconstructions of lower quality than those in the transmittance configuration. When the transmittance configuration is considered the $\mathrm{CW}$ and the TR reconstructions are visually indistinguishable and, as a result, the TR information is of limited interested. This behavior has already been reported in [24]. As pointed out in the introduction, however, the reflectance configuration can be the only one available for some applications, as for instance in brain function imaging, prostate tomography, or tissue oxymetry. In this configuration, on the contrary, the TR reconstruction quality is observed to outperform the $\mathrm{CW}$ reconstruction quality. 

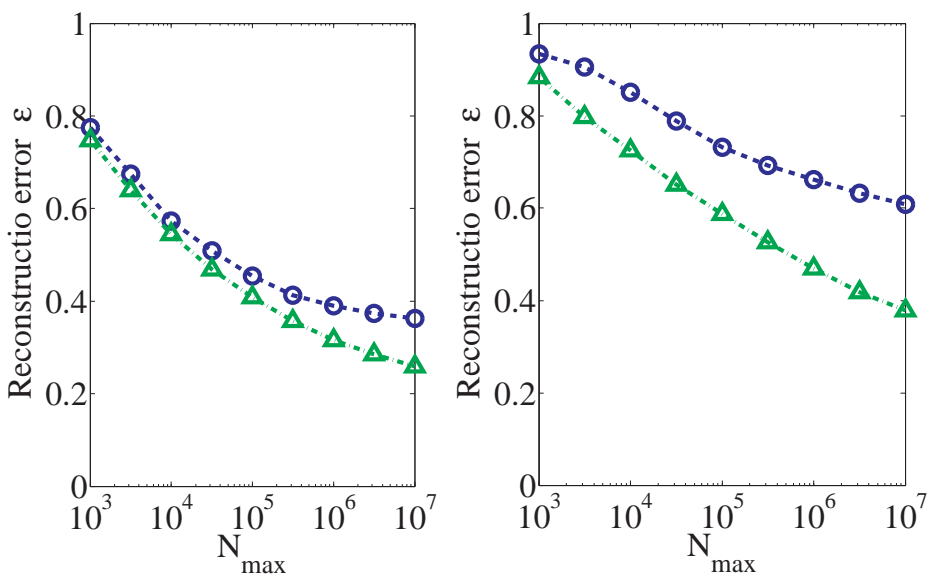

Figure 5: Reconstruction error $\mathcal{E}$ as a function of the maximum number of detected photons for both CW modality (blue line marked with $\bigcirc$ ) and TR modality (green line marked with $\triangle$ ). Results for the transmittance configuration are plotted on the left while results for the reflectance configuration are plotted on the right.

Indeed, the inclusions 1 and 2 seem to be better localized and quantified. The inclusion 3, which is not present in the CW reconstruction, is brought to light using the TR approach.

To test these visual findings, we evaluated a global performance metric, namely the reconstruction error $\mathcal{E}$ defined by Eq. (12). Its value is reported in Fig. 5 as a function of the maximum number of detected photons $N_{\max }$ ranging from $10^{3}$ to $10^{7}$ photons. Both reflectance and transmittance configurations are investigated. Whatever the source-detector configuration, the reconstruction error is found to decrease for increasing $N_{\text {max }}$, illustrating the improvement of the reconstruction quality when measurements with larger signal-to-noise ratio are considered. For a given $N_{\max }$, the reconstruction error confirms the previous observations. In the transmittance configuration, the $\mathrm{CW}$ and TR reconstruction errors are very close in value and almost identical for $N_{\max }<10^{5}$ photons. On the contrary, the reconstructions in the reflectance configuration show that TR reconstruction error is significantly smaller than the $\mathrm{CW}$ reconstruction error even for $N_{\max }$ as small as $3.10^{4}$ photons.

Last, we evaluate for each of the three inclusions two local metrics, namely the localization error $\mathcal{L}$ and the quantification $Q$ defined in Eq. (13) and Eq. (14), respectively. The results in the transmittance configuration are depicted in Fig. 6 while the reflectance results are depicted in Fig. 7.

As far as the transmittance is concerned, the TR quantification and localization error slightly differ from the CW quantification and localization error. The benefit of the TR approach is limited to the quantification, for $N_{\max }$ over $10^{5}$ photons, and in areas close to the source or detector planes (see inclusion 1 and 3). For $N_{\max }<10^{5}$, photons difference between $\mathrm{CW}$ and TR for both localization error and quantification are hardly noticeable, which is in agreement with our previous findings as well as those reported in [24].

As far as the reflectance is concerned, both localization error and quantification are improved when the TR approach is used rather than the $\mathrm{CW}$ approach. It can be seen that the TR quantification is overestimated in surface (see of inclusion 1) for large $N_{\text {max }}$. Yet, for all the other cases, the quantification of the CW approach is lower than that of the TR approach (see inclusions 2 and 3, notably). The same trend is observed with regard to the localization error of the three inclusions, which is always found to be better in the TR case than in the CW base. The observed oscillations in the value of the localization error in Fig. 6 can be mainly explained by numerical errors occurring when the localization error tends to zero. A substantial improvement is observed for inclusion 3, which is located far away from the source-detector plane in a zone where the $\mathrm{CW}$ exhibits poor performances.

\section{Conclusion}

In this work we addressed the problem of fluorescence diffuse optical tomography and we compared the performances of the time-resolved and continuous-wave approaches. Three different merit parameters for the quality of the reconstructions, namely reconstruction error, localization error and quantification, have been regarded. We observed that the reconstruction quality of a fluorescent inclusion embedded in a highly scattering media can be improved employing the time-resolved approach, even in realistic scenarios for which measurement noise and limited dynamic range are considered. Therefore our results indicate that the time-resolved information is more beneficial in the reflectance configuration than in the transmittance configuration.

\section{Conflicts of interest statement}

There is no conflict of interest.

\section{Acknowledgment}

This work was supported in part by CARIPLO Foundation (grant 2009-2626). 

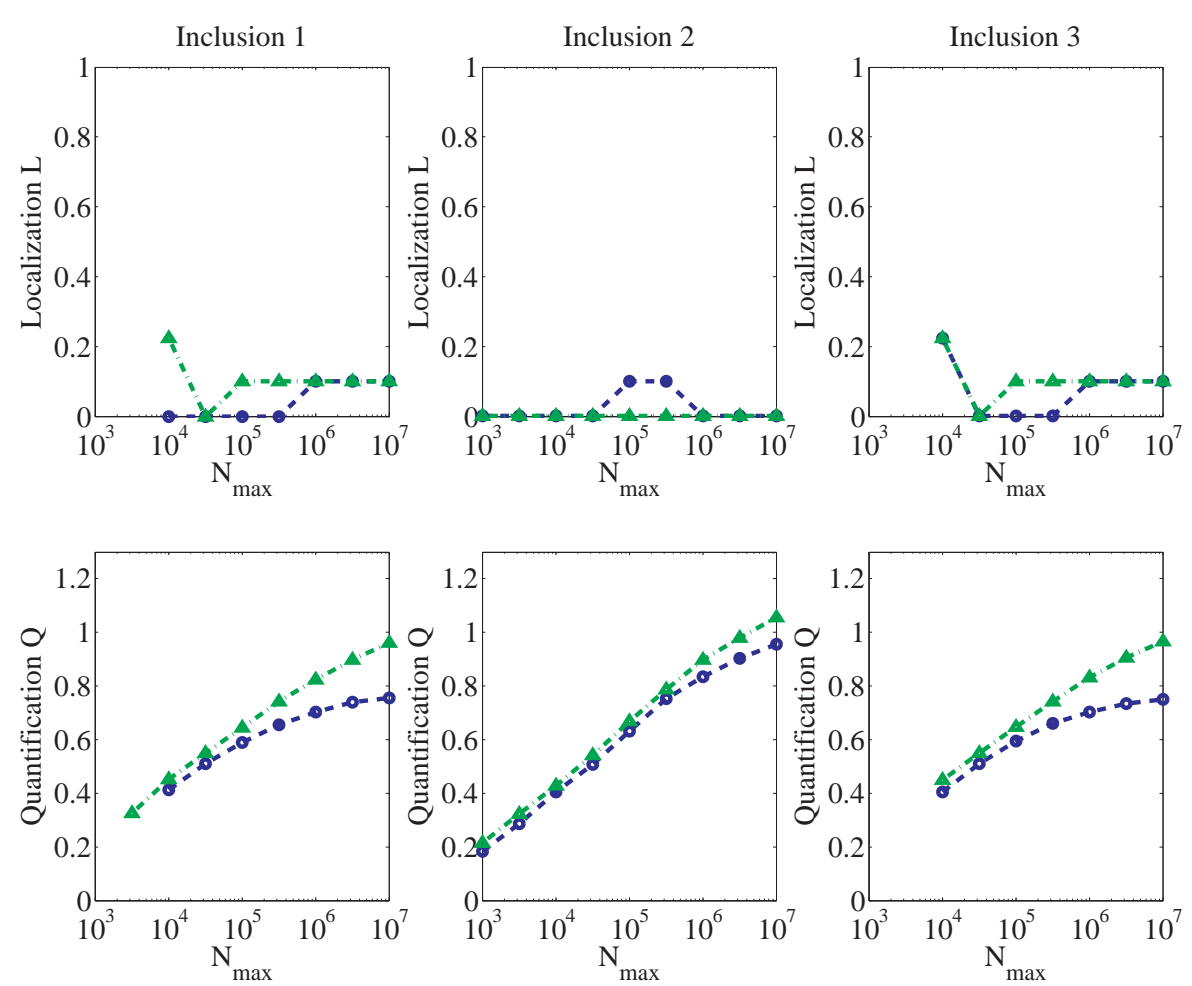

Figure 6: Reconstruction performances in the transmittance configuration for both $\mathrm{CW}$ modality (blue line marked with $\bigcirc$ ) and TR modality (green line marked with $\triangle$ ). The localization error $\mathcal{L}$ is represented in the top row, the quantification $Q$ in the bottom row. Inclusion 1 is considered in the left-hand column, inclusion 2 in the middle column, inclusion 3 in the right-hand column.
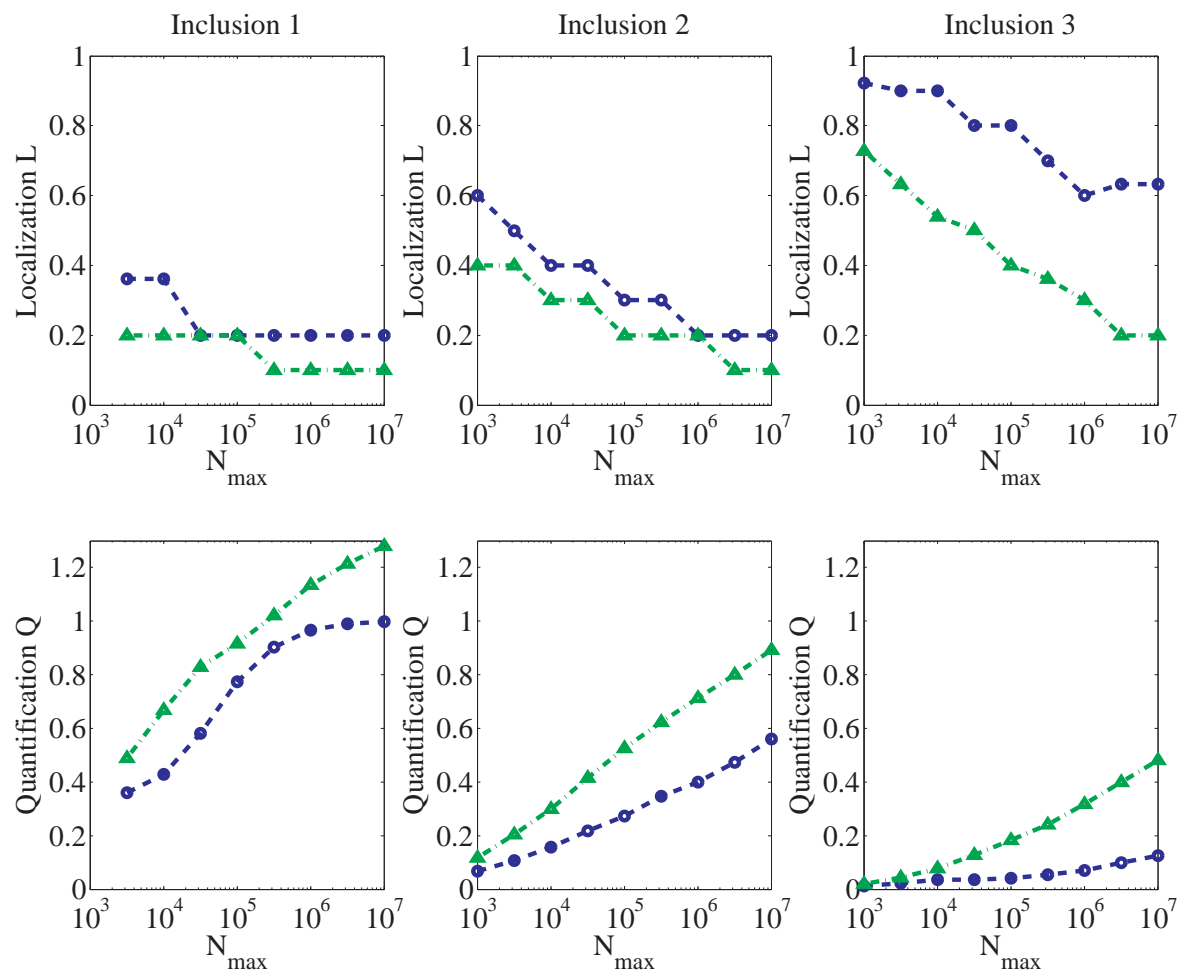

Figure 7: Reconstruction performances in the reflectance configuration for both CW modality (blue line marked with $\bigcirc$ ) and TR modality (green line marked with $\Delta$ ). The localization error $\mathcal{L}$ is represented in the top row, the quantification $Q$ in the bottom row. Inclusion 1 is considered in the left-hand column, inclusion 2 in the middle column, inclusion 3 in the right-hand column. 


\section{References}

[1] A. Yodh, B. Chance, Spectroscopy and imaging with diffusing light, Physics Today 48 (3) (1995) 34-40.

[2] D. A. Boas, D. H. Brooks, E. L. Miller, C. A. DiMarzio, M. Kilmer, R. J. Gaudette, Q. Zhang, Imaging the body with diffuse optical tomography, IEEE Signal Processing Magazine 18 (6) (2001) 57-75. URL $<$ Go to ISI $>$ : //000171836800008

[3] A. P. Gibson, J. C. Hebden, S. R. Arridge, Recent advances in diffuse optical imaging, Physics in Medicine and Biology 50 (4) (2005) R1-R43. URL http://stacks . iop.org/0031-9155/50/R1

[4] D. Y. Paithankar, A. U. Chen, B. W. Pogue, M. S. Patterson, E. M. SevickMuraca, Imaging of fluorescent yield and lifetime from multiply scattered light reemitted from random media, Appl. Opt. 36 (10) (1997) 22602272.

URL http: //ao.osa.org/abstract. cfm?URI=ao-36-10-2260

[5] V. Ntziachristos, C.-H. Tung, C. Bremer, R. Weissleder, Fluorescence molecular tomography resolves protease activity in vivo, Nat Med 8 (7) (2002) 757-761.

URL http://dx.doi.org/10.1038/nm729

[6] L. Hervé, A. Koenig, A. Da Silva, M. Berger, J. Boutet, J. Dinten, P. Peltié, P. Rizo, Noncontact fluorescence diffuse optical tomography of heterogeneous media, Applied Optics 46 (22) (2007) 4896-4906.

[7] C. Bremer, V. Ntziachristos, R. Weissleder, Optical-based molecular imaging: contrast agents and potential medical applications, European Radiology 13 (2) (2003) 231-243.

URL ht tp://dx. doi .org/10.1007/s00330-002-1610-0

[8] I. L. Medintz, H. T. Uyeda, E. R. Goldman, H. Mattoussi, Quantum dot bioconjugates for imaging, labelling and sensing, Nat Mater 4 (6) (2005) 435-446.

URL http://dx.doi.org/10.1038/nmat 1390

[9] V. Ntziachristos, A. H. Hielscher, A. G. Yodh, B. Chance, Diffuse optical tomography of highly heterogeneous media, Ieee Transactions on Medical Imaging 20 (6) (2001) 470-478.

URL $<$ Go to ISI $>$ : //000169452900002

[10] R. Roy, A. Godavarty, E. M. Sevick-Muraca, Fluorescence-enhanced optical tomography using referenced measurements of heterogeneous media, Ieee Transactions on Medical Imaging 22 (7) (2003) 824-836. URL <Go to ISI $>$ : //000184443900004

[11] E. E. Graves, J. Ripoll, R. Weissleder, V. Ntziachristos, A submillimeter resolution fluorescence molecular imaging system for small animal imaging, Medical Physics 30 (5) (2003) 901-911. doi:10.1118/1.1568977. URL ht tp: //link. aip.org/link/?MPH/30/901/1

[12] A. Koenig, L. Herve, V. Josserand, M. Berger, J. Boutet, A. D. Silva, J.M. Dinten, P. Peltie, J.-L. Coll, P. Rizo, In vivo mice lung tumor followup with fluorescence diffuse optical tomography, Journal of Biomedical Optics 13 (1) (2008) 011008. doi:10.1117/1.2884505. URL ht tp://link. aip.org/link/?JBO/13/011008/1

[13] G. Yu, T. Durduran, D. Furuya, J. H. Greenberg, A. G. Yodh, Frequencydomain multiplexing system for in vivo diffuse light measurements of rapid cerebral hemodynamics, Appl. Opt. 42 (16) (2003) 2931-2939. URL http: //ao.osa.org/abstract.cfm?URI=ao-42-16-2931

[14] S. Lam, F. Lesage, X. Intes, Time domain fluorescent diffuse optical tomography: analytical expressions, Optics Express 13 (7) (2005) 2263 2275

[15] A. Liebert, H. Wabnitz, H. Obrig, R. Erdmann, M. Moller, R. Macdonald, H. Rinneberg, A. Villringer, J. Steinbrink, Non-invasive detection of fluorescence from exogenous chromophores in the adult human brain, Neuroimage 31 (2) (2006) 600-608.

[16] F. Gao, H. J. Zhao, Y. Tanikawa, Y. Yamada, A linear, featured-data scheme for image reconstruction in time-domain fluorescence molecular tomography, Optics Express 14 (16) (2006) 7109-7124.

[17] A. Laidevant, A. D. Silva, M. Berger, J. Boutet, J.-M. Dinten, A. C. Boccara, Analytical method for localizing a fluorescent inclusion in a turbid medium., Appl Opt 46 (11) (2007) 2131-2137.

[18] J. Riley, M. Hassan, V. Chernomordik, A. Gandjbakhche, Choice of data types in time resolved fluorescence enhanced diffuse optical tomography, Medical Physics 34 (2007) 4890.

[19] N. Ducros, L. Herve, A. D. Silva, J.-M. Dinten, F. Peyrin, A comprehensive study of the use of temporal moments in time-resolved diffuse optical tomography: part i. theoretical material, Physics in Medicine and Biology
54 (23) (2009) 7089-7105. doi:10.1088/0031-9155/54/23/004.

URL http://stacks . iop . org/0031-9155/54/7089

[20] N. Ducros, A. D. Silva, L. Herve, J.-M. Dinten, F. Peyrin, A comprehensive study of the use of temporal moments in time-resolved diffuse optical tomography: part ii. three-dimensional reconstructions, Physics in Medicine and Biology 54 (23) (2009) 7107-7119. doi:10.1088/0031$9155 / 54 / 23 / 005$

URL http://stacks . iop.org/0031-9155/54/7107

[21] S. R. Arridge, W. R. B. Lionheart, Nonuniqueness in diffusion-based optical tomography, Opt. Lett. 23 (11) (1998) 882-884.

URL ht tp: //ol . osa.org/abstract. cfm?URI=01-23-11-882

[22] A. T. N. Kumar, S. B. Raymond, A. K. Dunn, B. J. Bacskai, D. A. Boas, A time domain fluorescence tomography system for small animal imaging, Medical Imaging, IEEE Transactions on 27 (8) (2008) 1152-1163. doi:10.1109/TMI.2008.918341.

[23] J. Selb, A. Dale, D. Boas, Linear 3d reconstruction of time-domain diffuse optical imaging differential data: improved depth localization and lateral resolution, Optics Express 15 (25) (2007) 16400-16412.

[24] N. Ducros, A. D. Silva, J.-M. Dinten, C. S. Seelamantula, M. Unser, F. Peyrin, A time-domain wavelet-based approach for fluorescence diffuse optical tomography, Medical Physics 37 (6) (2010) 2890-2900. doi:10.1118/1.3431571. URL ht tp://link. aip.org/link/?MPH/37/2890/1

[25] S. R. Arridge, J. C. Schotland, Optical tomography: forward and inverse problems, Inverse Problems 25 (12) (2009) 123010 (59pp). URL http: //stacks . iop.org/0266-5611/25/123010

[26] M. S. Patterson, B. W. Pogue, Mathematical-model for time-resolved and frequency-domain fluorescence spectroscopy in biological tissue, Applied Optics 33 (10) (1994) 1963-1974. URL $<$ Go to ISI $>$ : //A1994ND42400024

[27] N. Ducros, A. da Silva, J.-M. Dinten, F. Peyrin, Approximations of the measurable quantity in diffuse optical problems: theoretical analysis of model deviations, J. Opt. Soc. Am. A 25 (5) (2008) 1174-1180.

[28] A. Bassi, C. D’Andrea, G. Valentini, R. Cubeddu, S. Arridge, Temporal propagation of spatial information in turbid media, Opt. Lett. 33 (23) (2008) 2836-2838.

URL http://ol .osa.org/abstract . cfm?URI=01-33-23-2836

[29] V. Lukic, V. A. Markel, J. C. Schotland, Optical tomography with structured illumination, Opt. Lett. 34 (7) (2009) 983-985.

URL ht tp: //ol . osa.org/abstract . cfm?URI=ol -34-7-983

[30] C. D'Andrea, N. Ducros, A. Bassi, S. Arridge, G. Valentini, Fast 3d optical reconstruction in turbid media using spatially modulated light, Biomed. Opt. Express 1 (2) (2010) 471-481. URL http: //www . opt icsinf obase . org/boe/abstract. cfm?URI=boe-1-2-

[31] N. Ducros, C. D'andrea, G. Valentini, T. Rudge, S. Arridge, A. Bassi, Full-wavelet approach for fluorescence diffuse optical tomography with structured illumination, Opt. Lett. 35 (21) (2010) 3676-3678. URL ht tp://ol .osa.org/abstract . cfm?URI=01-35-21-3676

[32] [link]. URL http://web4.cs.ucl.ac.uk/research/vis/toast/

[33] A. Pifferi, A. Torricelli, P. Taroni, D. Comelli, A. Bassi, R. Cubeddu, Fully automated time domain spectrometer for the absorption and scattering characterization of diffusive media, Review of Scientific Instruments 78 (5) (2007) 053103. doi:10.1063/1.2735567. URL ht tp://link. aip.org/link/?RSI/78/053103/1 